\title{
Universal health informatics competencies for working clinicians in the UK: where are we now and where should we be going? A mixed methods study
}

\author{
Authors: Lydia Jidkov, ${ }^{\mathrm{A}}$ Amitava Banerjee ${ }^{\mathrm{A}, \mathrm{B}}$ and Pippa Bark ${ }^{\mathrm{A}}$
}

\author{
Aims \\ To map health informatics (HI) competencies in UK postgraduate \\ medical education.
}

\section{Methods}

Scoping review, content analysis of postgraduate clinician training curricula and expert consultation to validate findings and identify key HI competencies. The International Medical Informatics Association (IMIA) recommendations for biomedical and health informatics education were used to develop a framework of competency domains. This was termed the IMIA+ framework and was used for competency mapping including information such as suggested competency skill level (core, standard, specialist), target population and dissemination environment. The scoping review search was conducted using PubMed electronic database on and up to 10 March 2017. Documents were eligible for inclusion if they described detailed postgraduate HI competencies that specified clinicians within their target population. Search terms were peer-reviewed and all article types in the English language were included. Date range 1998-present was searched to limit outdated information but capture documents following the introduction of the National Programme for Information Technology. Postgraduate clinician training curricula were sourced from the General Medical Council website on 11 May 2017 and searched for peer- reviewed keywords: informatic*, technolog*, 'IT system', 'electronic [health/medical/clinical/patient] record' and 'e[-]Health'. Expert consultation consisted of face-to-face and telephone interviews between 1 June and 6 July 2017.

\section{Results}

Twenty-one scoping review documents were mapped to 45 of 48 IMIA+ framework domains, including three additional domains identified from the documents. Core competencies identified in review documents did not correlate directly with introductory domains in the IMIA framework. Sixty-eight curricula documents

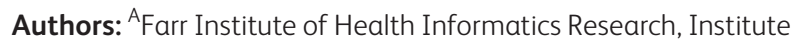
of Health Informatics, University College London, London; ${ }^{\mathrm{B}}$ Health Informatics Unit, Royal College of Physicians, London, UK were identified and mapped across 29 of 50 IMIA+ framework domains (two additional curricula specific domains were added at this stage). Training curricula mapped between zero domains (ophthalmology and core surgical training) and 16 domains (pathology). Experts validated that universal $\mathrm{HI}$ competencies were vital for UK clinicians but were underrepresented in current curricula. The experts gave insight into missing and required universal competencies under the topic areas: information governance and security, information and knowledge management, secondary use of data, digital communication, system and staff safety, and patient empowerment and emerging technologies. Experts emphasised the importance of integrating $\mathrm{HI}$ competencies throughout curricula as opposed to a standalone set of competencies.

\section{Conclusion}

Postgraduate medical education neglects universal HI competencies in the UK. This study has identified key competencies for integration into training curricula which are vital in the context of digitisation efforts within the NHS.

\section{Conflict of interest statement}

None to declare. 